\title{
Via and Embedded Resistor Production in Low Cost Lithographically Printed Substrates
}

\author{
J.M. Gilbert ${ }^{1}$, G.R. Elliner ${ }^{1}$, P.H. Key ${ }^{2}$, H.V. Snelling ${ }^{2}$, D. Harrison ${ }^{3}$ and P. Harrey ${ }^{3}$
}

1 School of Engineering

University of Hull

Hull, HU6 7RX, UK

Tel: +441482466379

Fax: +441482466664

email: j.m.gilbert@eng.hull.ac.uk
2 Department of Physics

University of Hull

Hull, HU6 7RX, UK
3 Department of Design Brunel University

Runnymead Campus

Egham, Surrey

TW20 0JZ, UK

\begin{abstract}
Lithographic printing of silver based conductive inks to form circuit tracking provides a very low cost, environmentally friendly method of production. It allows circuits with line widths down to $100 \mu \mathrm{m}$ and a sheet resistance of $0.3 \Omega$ per square to be produced at rates of up to 10000 impressions per hour. The process makes use of low capital cost equipment and has low set up costs. To date, an equally cost effective method of manufacturing vias in these circuits has not been developed. This paper proposes two new techniques for via production which are compatible with the rest of the manufacturing process. In the first technique, suitably profiled holes are laser drilled for subsequent filling as part of the lithographic printing. In the second technique laser energy is used to melt the substrate material so that it is able to mix with the conductive ink and so form a conductive path. The paper describes the proposed production techniques and discusses the factors affecting each method and in particular the substrate properties affecting production. The substrate materials considered in detail are Glossart and Teslin. The optical absorption and ablation characteristics of these materials for excimer and $\mathrm{CO}_{2}$ lasers are reported, as are the thermal diffusivities.
\end{abstract}

\section{Lithographic Printing of Circuit Substrates}

Conductive Lithographic Films (CLFs) are a recently developed means of producing low cost substrates without many of the negative environmental consequences of conventional processes [1]. It is a purely additive process, thereby avoiding the expensive and potentially polluting photo resist and etching processes.

The offset lithographic process adopted makes use of a printing plate cylinder on which the image is initially formed and a blanket cylinder which transfers the image onto the substrate which is itself carried on the impression cylinder. The printing plate cylinder passes a moistening roller followed by an inking roller. The chemistry of the printing plate cylinder repels water in areas where the image is dark. This allows the oil based ink transferred from the inking roller to adhere in these areas. Those areas of the printing plate cylinder which are to remain light are hydroscopic and so pick up water from the moistening roller and hence repel the ink when passing the ink rollers. The image formed on the printing plate cylinder is transferred to the blanket cylinder and thence to the substrate. Using commercial printing equipment, printing can be carried out at up to 10000 impressions per hour. Once applied to the substrate a curing period is required to allow evaporation of the carrier elements in the ink and/or oxidisation of the ink's conductive particles. This can be carried out at room temperature or can be accelerated by the use of elevated temperature.

A critical element in the development of the lithographic technique is the formulation of an ink which has both good printing characteristics and, when cured, sufficiently low resistivity. The latter point is particularly relevant when it is considered that the typical thickness of a lithographically printed image is only $3 \mu \mathrm{m}$ compared to around $50 \mu \mathrm{m}$ for screen printed inks and similar thicknesses used in conventional copper laminate boards. The inks developed use silver particles in an organic resin carrier and these have been engineered to be compatible with a range of substrate materials while maintaining rheology suitable for the printing process. Using these inks and commercial printing equipment allow minimum track and gap distances of around $100 \mu \mathrm{m}$. Although impressions can be made of smaller features, the need to overprint to ensure consistent conductivity, means that registration errors prevent these finer features from being used practically.

Once cured the ink has a sheet resistance of around $0.3 \Omega$ per square. This is a factor of approximately ten times higher than screen printed inks. This resistivity does limit the circuits to low power and moderate speed applications but they have been used in a range of microprocessor, telecommunication and microwave circuits [2].

\section{Via and Resistor Formation Methods}

To be a practical form of circuit substrate, even for moderate levels of circuit density, it is crucial that a means of producing vias between the two conducting layers on a double sided substrate be available. It is also important that the technique be compatible with the remainder of the process. In particular, the vias should be of similar dimensions to the printed tracking and be produced at low cost without adverse environmental consequences and, if possible, at high production rates.

Clearly methods used for via production in conventional circuit boards are not compatible with lithographically printed substrates since the processes involved would cause damage to both the substrate and the printed ink. To date, the method adopted has been to mechanically drill holes and then manually fill these with conductive adhesive. Clearly this is not viable for volume production. It may be possible that the filling process could be carried out as part of the screen 
printing process which is used to deposit conductive adhesive for component attachment but this has not been tested. Although this method does allow vias to be produced, it has a number of disadvantages. The use of mechanical drilling limits the size of via holes to be above approximately $0.5 \mathrm{~mm}$. It is anticipated that difficulties may be encountered in ensuring that the screen printed ink reaches the full depth of the via and forms a reliable connection with the underside tracking. In addition, the adhesive curing process would not allow sufficient time after via production for testing prior to population if adhesive were applied for both vias and attachment simultaneously.

The two novel techniques described in this paper aim to overcome these difficulties without the introduction of excessive cost or additional process step through the use of controlled laser energy. The techniques are outlined in the following sections.

\section{Laser Drill and Fill}

An obvious alternative to mechanical drilling to produce via holes is to use laser drilling. Such holes may then be filled with screen printed conductive adhesive as previously described. Alternatively, if via holes are produced in the substrate prior to lithographic printing then it may be anticipated that some of the ink would be forced into the via holes during the printing process. If sufficient ink enters the hole then a conductive link could be formed.

The use of laser drilling offers advantages over other techniques whichever filling method is adopted. Laser drilling allows finer features to be produces but it also offers greater flexibility over hole profile. The use of a tapered hole may result in more reliable filling than a straight, mechanically drilled, hole would give. If an imaged laser beam is used then a circuit specific contact mask is not required, unlike in plasma etching techniques.

\section{Melt and Flow}

By projecting the laser onto the printed surface at a fluence below the ablation threshold it may be possible to melt the substrate and so allow the silver ink particles to mix with the substrate. This heating may also encourage conducting particles to migrate into the substrate. Provided sufficient ink particles are incorporated, a conductive link may be formed. It has been shown in previous work that this techniques can be used to form vias in screen printed substrates but screen printing generally deposits a greater volume of ink than lithographic printing and so the amount of ink available to form a link is higher. This work also showed that a second mechanisms is important in the via formation in addition to simple melting and mixing. During heating some of the substrate material is vaporised and released, reducing the overall volume and allowing the two surfaces to move closer together making link formation easier.

Clearly, a technique which relies on the substrate melting and flowing together with the ink is only feasible for substrate materials which can be melted but conducting particle migration may still be possible in non-melting substrates.

\section{Resistor Formation}

Both of the techniques described above may be adaptable to the production of embedded resistors. In the case of drilled vias, by printing a layer of resistive ink into the via hole a resistor may be formed while in the melt and flow method, careful control of the conductor distribution may allow resistive elements to be formed. It is anticipated that such resistors would only be suitable for low precision applications.

\section{Substrate Material and Their Properties}

Several materials have been found to offer an acceptable combination of printing performance and electrical properties for use as substrates. Of these, two will be considered here.

Glossart is formed of a standard paper base coated with a fine clay to produce a smoother surface than untreated paper but retaining sufficient surface roughness to allow good ink adhesion. Teslin is formed from silica spheres suspended in a polyolefin carrier. Once formed into sheets the majority of the carrier is dissolved leaving air pockets as a substantial proportion of the sheet volume.

In order to understand the processes of via formation using the proposed techniques, a number of material characteristics must be understood. In particular, the choice of laser to give the best performance for laser drilling or laser heating depends on the optical absorption of the materials and their thermal diffusivity.

The optical absorption depth of a material, for a particular laser wavelength, is $1 / \alpha$ where $\alpha$ is the absorption coefficient. This can be measured for small signals by transmission spectra. During the laser pulse, this depth of heated material may be extended due to thermal diffusion. The total laser heated depth, $h$, can be found from $h=\frac{1}{\alpha}+\sqrt{2 D \tau}$ where $\mathrm{D}$ is the thermal diffusivity and $\tau$ the laser pulse duration. Thus the thermal diffusivity and absorption coefficient are important parameters in determining the expected etch rates. In particular, $\alpha$ must be considered when selecting a laser/material combination.

\section{Optical Absorption Measurement}

The IR optical transmission spectra of $95 \mu \mathrm{m}$ Glossart and $205 \mu \mathrm{m}$ Teslin sheets have been measured. It was found that Glossart has $>99 \%$ absorption across the entire $200 \mathrm{~nm}$ to $15 \mu \mathrm{m}$ wavelength range and so will absorb optical energy from any laser operating in this range. Teslin is also highly absorbing around the $10.6 \mu \mathrm{m}$ range at which the $\mathrm{CO}_{2}$ laser operates but is slightly less absorbing at other wavelengths with a transmissivity of up to $20 \%$ at shorter wavelengths. The absorption depth for a particular wavelength laser can be determined from the short pulse etch rate. Values determined using this method are given in Table 1 for the materials and lasers considered here. Due to the relatively long wavelength of the $\mathrm{CO}_{2}$ laser it has a large absorption depth, $\left(\alpha^{-1}\right)$. Although silica itself is transmissive around the shorter wavelength $(248 \mathrm{~nm})$ of the ArF excimer laser the internal structure of Teslin results in scattering effects which lead to a lower absorption depth. 


\begin{tabular}{|l|c|c|}
\cline { 2 - 3 } \multicolumn{1}{c|}{} & \multicolumn{2}{c|}{ Laser Type } \\
\hline Material & Excimer (248nm) & $\mathrm{CO}_{2}$ \\
\hline Glossart & - & $32.4 \mu \mathrm{m}$ \\
\hline Teslin & $1.3 \mu \mathrm{m}$ & $30.0 \mu \mathrm{m}$ \\
\hline
\end{tabular}

Table 1 Absorption Depth in Glossart and Teslin for $\mathrm{CO}_{2}$ and Excimer Lasers

\section{Thermal Characteristics}

The thermal diffusivity of a material can be determined using an adaptation of the technique described in [5] which involves the application of a pulse of laser energy to one side of a sheet of material while logging the temperature rise on the reverse side. Using this method the diffusivity figures for Glossart and Teslin have been determined as those given in

Table 2.

\begin{tabular}{|c|c|}
\hline Material & $\begin{array}{c}\text { Thermal Diffusivity } \\
\left(10^{-4} \mathrm{~cm}^{2} \mathrm{~s}^{-1}\right)\end{array}$ \\
\hline Glossart & 3.87 \\
\hline Teslin & 9.45 \\
\hline
\end{tabular}

Table 2 Thermal Diffusivity of Glossart and Teslin

The technique used to measure these values assumes that the material is homogeneous and although this is not strictly valid for either material it is believed that the effect of inhomogeneity is not insignificant.

\section{Ablation Characteristics}

The ablation rates for Glossart and Teslin using a TEA: $\mathrm{CO}_{2}$ laser at various fluences are shown in Figure 1. It can be seen that the ablation threshold for the Glossart is higher than that for Teslin and that the etch rate is higher in Teslin. Similar trials were carried out using an ArF excimer laser operating at $248 \mathrm{~nm}$. It was found that the ablation threshold in Teslin is significantly lower than for $\mathrm{CO}_{2}$ at around $0.6 \mathrm{Jcm}^{-2}$. This would be expected since the smaller absorption depth means that the effect of the laser energy is concentrated near to the surface. However, once the $\mathrm{CO}_{2}$ laser is significantly above its ablation threshold it gives a higher ablation rate because the longer wavelength affects material to a greater depth. For the Glossart, once the excimer laser had removed the clay coating, the fibrous interior could not be ablated but became desiccated to the point where the fibres crumbled, leaving a very poor quality hole.

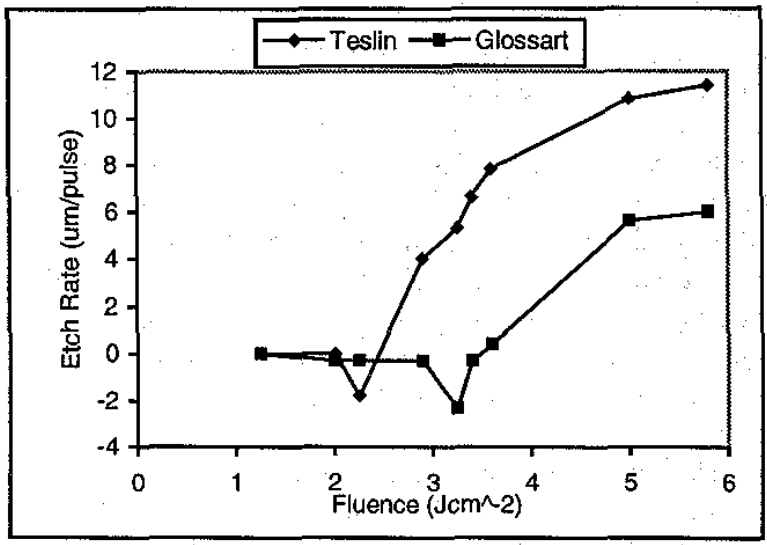

Figure 1 Mean Ablation Rate of Glossart and Teslin with TEA: $\mathrm{CO}_{2}$ Laser

An important feature of the $\mathrm{CO}_{2}$ laser ablation process in both materials was found to be that low fluence laser pulses do not result in ablation but instead cause a raising of the material surface. This can be seen in Figure 1 as a negative ablation rate at a fluence of $2.25 \mathrm{Jcm}^{-2}$ for Teslin and $3.25 \mathrm{Jcm}^{-2}$ for Glossart. This effect can be seen more clearly in Figure 2 which shows the surface profile across the centre of the Teslin ablation site following 1,5 and 10 laser pulses (each profile is offset to aid clarity).

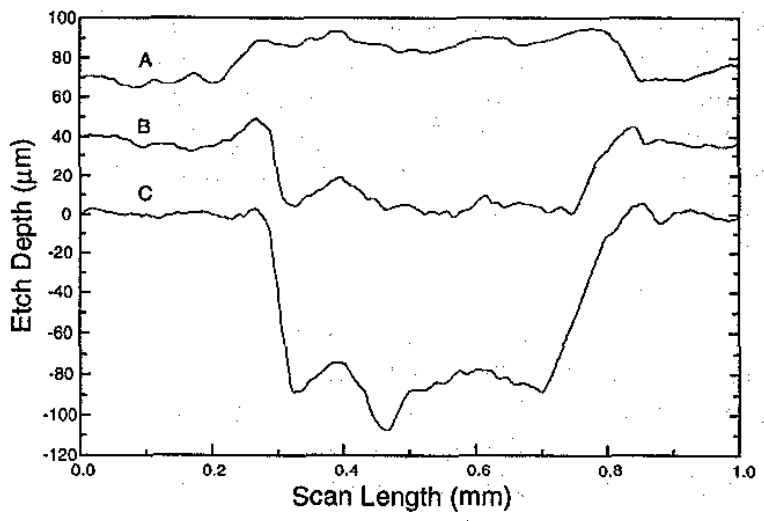

Figure 2 Ablation Site Profile after Successive Pulses A - 1 pulse, B - 5 pulses, C 10 - Pulses

Two mechanisms have been proposed to explain this effect. Heating of the air trapped inside the material may cause thermal expansion which lifts the surface where the laser energy is applied, and in a small surrounding area. Alternatively, the effect may be caused by expansion of the rapidly heated material near the surface. Since this cannot expand outwards, along the surface, it bulges upwards. Subsequent laser pulses remove most of the material but a remnant of this initial lifting remains in the presence of an annular lip around the hole with a height of around $5 \mu \mathrm{m}$. To date, this lip has not been found to cause difficulties in 
overprinting with the conductive ink but further analysis is required to ensure that there is no thinning of the ink deposit since this could cause excessive resistance.

\section{Practical Considerations in Via Formation}

A number of factors affect the way in which the proposed via production techniques must be realised. The most significant factors affecting each of the proposed schemes are discussed in the following sections.

\section{Laser Drill and Fill}

The surface quality of drilled via holes and the drilling rates which can be achieved are important as is the degree to which the hole profile can be controlled. Typical $\mathrm{CO}_{2}$ drilled via holes are shown in Figure 3 and Figure 4.

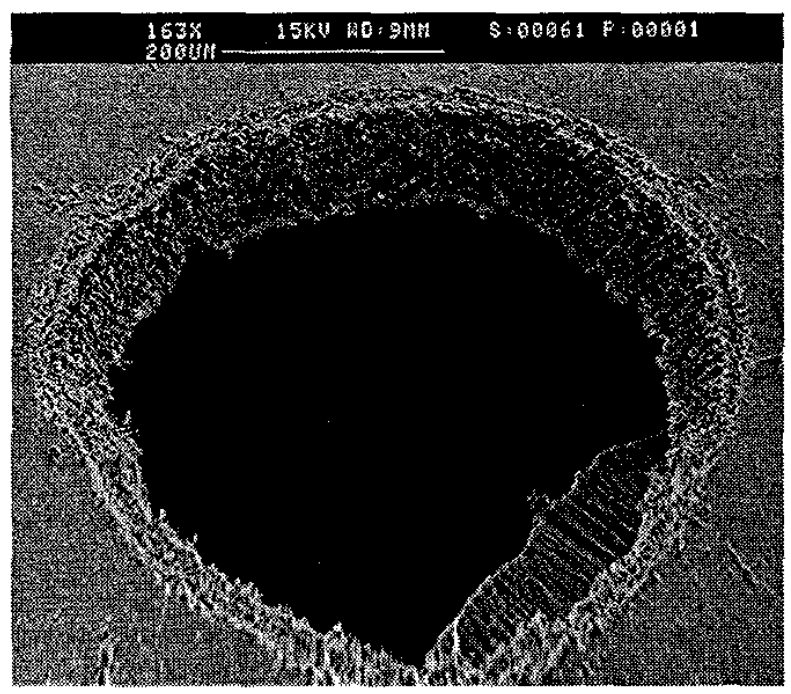

Figure $3 \mathrm{CO}_{2}$ Drilled Via Hole in Glossart

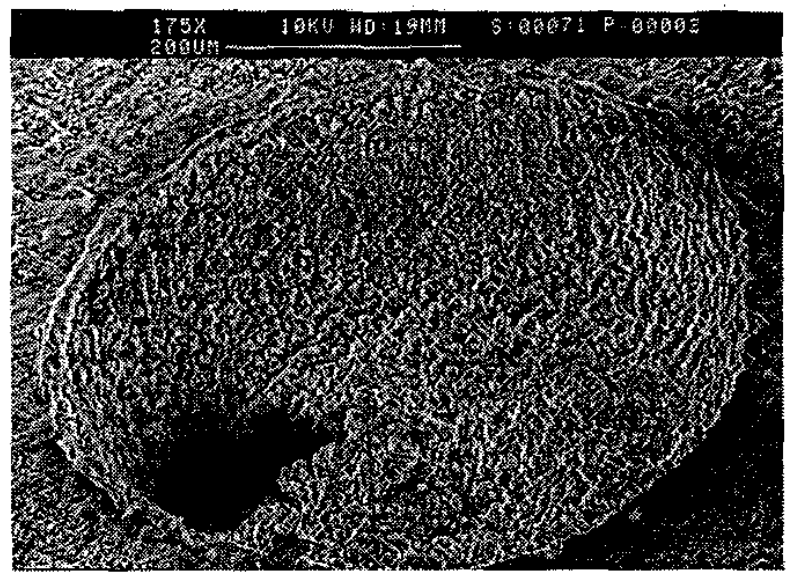

Figure 4 Partially Completed $\mathrm{CO}_{2}$ Drilled Hole in Teslin

These via holes may be compared with the excimer drilled holes shown in Figure 5 and Figure 6. On Glossart it can be seen that, at the modest fluence used the laser is unable to ablate the fibrous core of the material. In Teslin the blind hole formed with the excimer laser has much smoother sides and base than attained using $\mathrm{CO}_{2}$ and with edges which are more precisely defined.

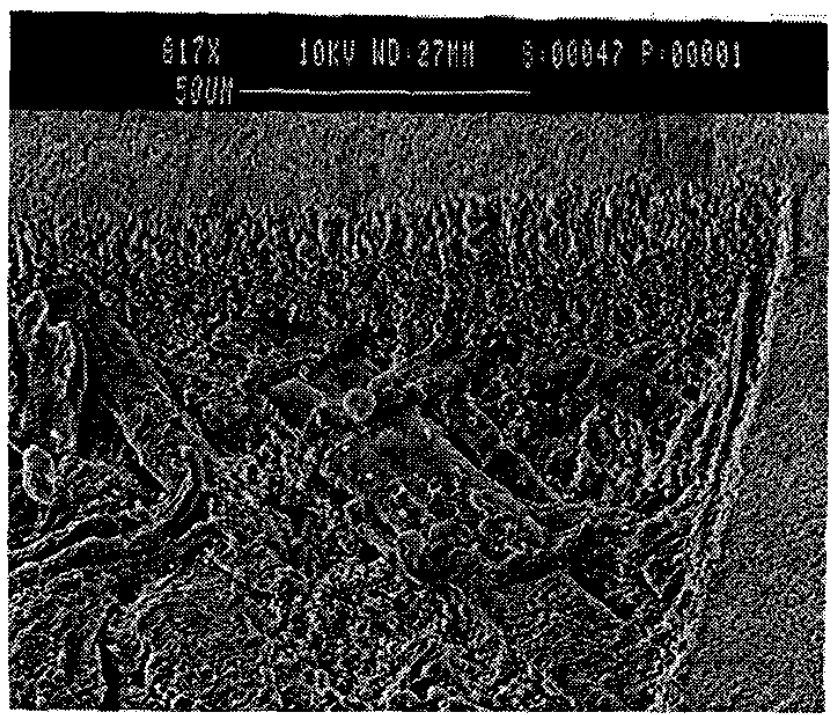

Figure 5 Excimer Ablation of Glossart Surface Coating

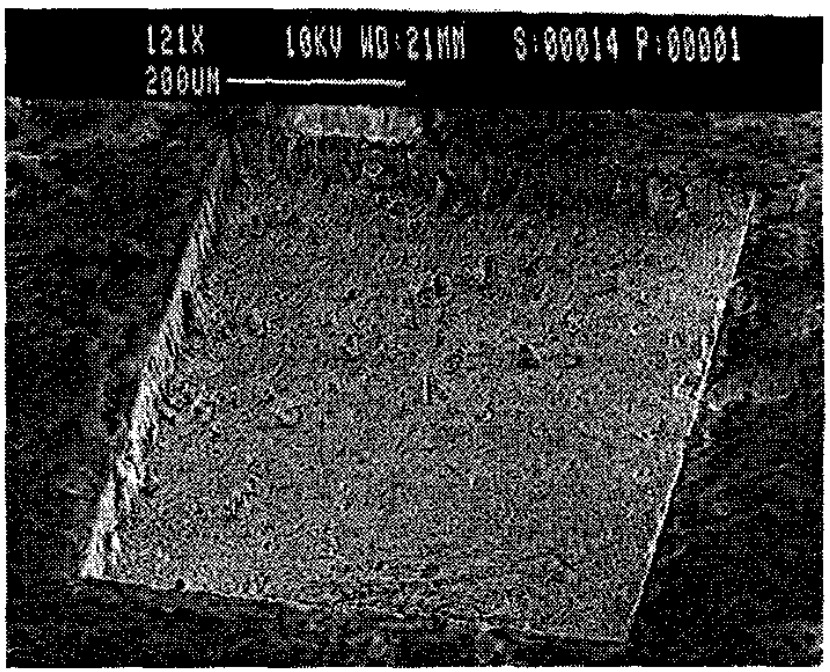

Figure 6 Excimer Laser Drilled Crater in Teslin

Print trials carried out on TEA: $\mathrm{CO}_{2}$ drilled via holes in both Glossart and Teslin have been carried out for $100 \mu \mathrm{m}$ and $200 \mu \mathrm{m}$ diameter holes. A typical sample is shown in

Figure 7. The presence of conductive ink in the interior of the via is suggested by the absence of any 'flaring' in the SEM image which would result from charge build up on insulating material. The presence of silver in small amounts was confirmed by the use of Energy Dispersion Analysis by X-ray (EDAX) equipment. However it has been found that although ink does enter the holes, it does not give a sufficiently consistent coating of the walls to provide a reliable current path. 


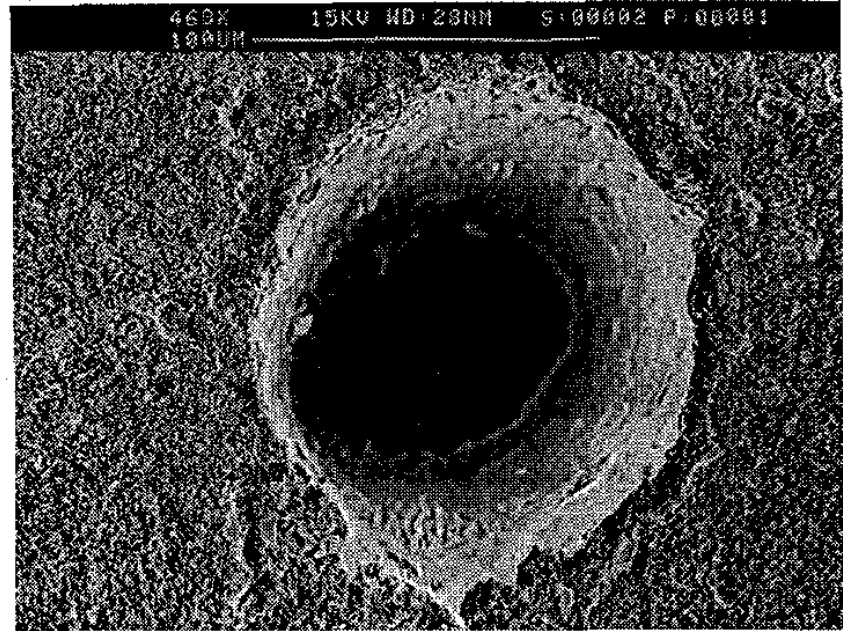

Figure $7 \mathrm{CO}_{2}$ drilled and Lithographically Printed Via

In order to improve ink flow into the via hole, the use of tapered profiles is currently being investigated. This may be achieved by reducing the laser spot diameter between successive pulses. An example of the resulting hole profile produced using $\mathrm{CO}_{2}$ and excimer lasers are shown in Figure 8 and Figure 9 respectively. In each case the laser spot size is reduced from approximately $600 \mu \mathrm{m}$ to $200 \mu \mathrm{m}$ in four linear steps.

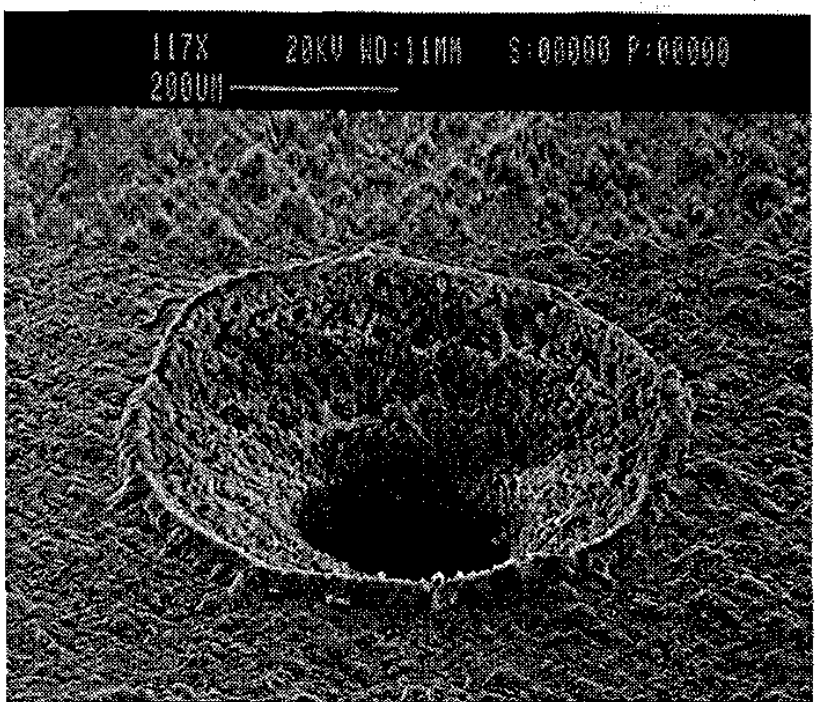

Figure 8 TEA:CO $\mathrm{CO}_{2}$ Laser Drilled Tapered Via Hole in Teslin

Once again, the excimer drilled hole has a more precisely defined shape than that produced using the $\mathrm{CO}_{2}$ laser as a result of the shorter wavelength of the excimer laser. The $\mathrm{CO}_{2}$ laser also produces a very pronounced lip but, as discussed previously, there is no evidence that this affects the ink filling behaviour of the via.

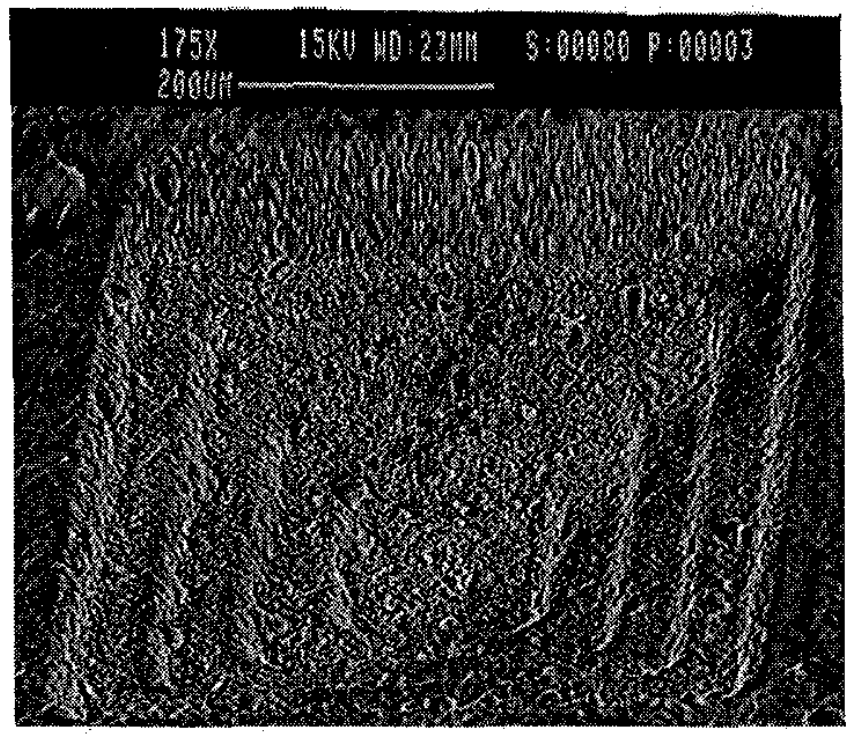

Figure 9 Excimer Drilled Tapered Via in Teslin

The resistance of the via connection is clearly an important consideration. From a simple analysis of the geometry it can be calculated that the total volume of ink which would be applied to the upper and lower surfaces of the via hole is $2 \pi r^{2} t_{i}$ where $\mathrm{r}$ is the via radius and $\mathrm{t}_{\mathrm{i}}$ the ink thickness. If all of this ink were applied uniformly to the side walls of the via the ink thickness, $t_{v}$, would be $t_{v}=t_{i} \frac{r}{d}$ where $\mathrm{d}$ is the substrate thickness. If the ink sheet resistance, when printed to a thickness of $t_{i}$, is $\rho$ and assuming that the resistance is proportional to the ink thickness, the resistance of such a via would be $R=\rho \frac{d^{2}}{2 \pi r^{2}}$. As the substrate thickness increases the ink is spread more thinly on the side walls and the current path becomes longer. As the via radius decreases the ink thickness reduces and the circumference of the inside of the via also reduces thus increasing its resistance.

In assessing the significance of this resistance it should be considered that a typical track may be $10 \mathrm{~mm}$ long and $100 \mu \mathrm{m}$ wide, thus having a resistance of $100 \rho$. The via resistance then becomes comparable if $\frac{d}{r} \geq 10 \sqrt{2 \pi}$. Given typical substrate thicknesses and realisable via dimensions, this situation is not likely to be reached. However it must be noted that this analysis assumes that all of the ink available is uniformly applied to the via walls. This is unlikely to be the case in practice and any other ink distribution will result in a higher via resistance. If tapered via holes are used then it can be shown that the resistance is higher, for a given maximum hole diameter, than for a straight via with the same radius. The increase is however only of the order of a factor of two for moderate amounts of taper. It should be noted that the above analysis does not take account of current crowding effects on 
the surface around the via hole but these effects are likely to be small.

\section{Melt and Flow}

The requirements for the laser characteristics for the proposed melt and flow technique are significantly different to those for drilling. Clearly ablation of the substrate is undesirable but heating to the full depth of the substrate is required. This implies the use of a $\mathrm{CW}$ rather than a short pulsed laser and a laser with a large absorption depth, such as the $\mathrm{CO}_{2}$. Previous work on the use of a $\mathrm{CW} \mathrm{CO}_{2}$ laser to heat Melinex substrates screen printed with silver ink has shown that the technique is able to produce vias with resistances of under $1 \Omega$ but it also highlighted a number of difficulties. Figure 10 shows the surface of a via produced in $135 \mu \mathrm{m}$ thick Melinex screen printed on both sides with $10 \mu \mathrm{m}$ of silver based ink using a $15 \mathrm{~W}$ laser pulse of $500 \mathrm{~ms}$ duration. It can be seen that considerable cracking of the ink has occurred. It is believed that this is due to contraction of the substrate during cooling. It is also found that some of the vias are brittle due to crystallisation of the Melinex during solidification.

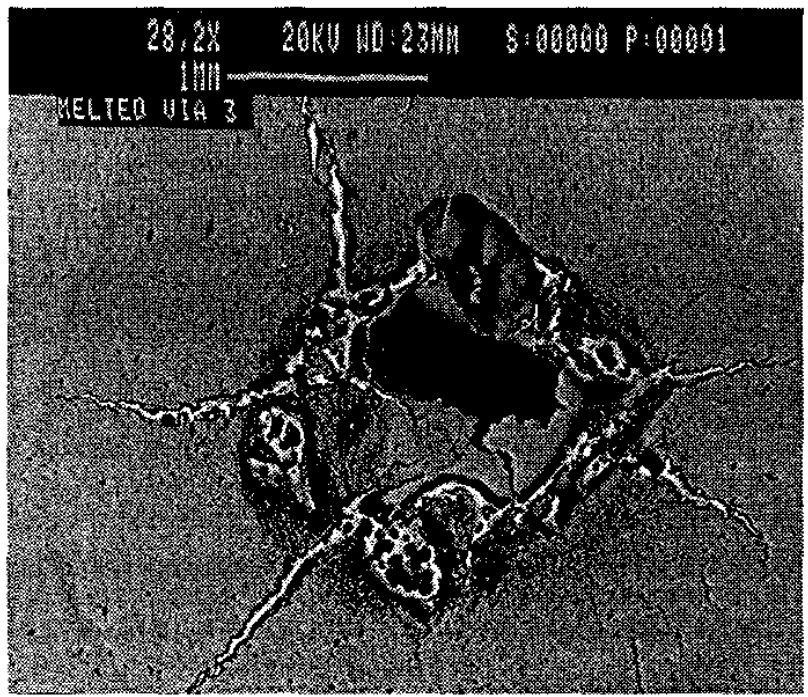

Figure 10 Melt and Flow Via in Screen Printed Melinex

When the substrate is heated several mechanisms can operate to allow the formation of a conducting path. A liquid pool in the substrate allows silver ink to fall through under the effect of gravity and so form a conduction path. Simultaneously, heating of the substrate will cause some of the more volatile components to be driven off, thus reducing the volume remaining in the area and bringing the two surfaces closer together.

In addition, mixing between ink and substrate will occur, resulting in a composite of conducting particles in an insulating matrix. A good deal of work has been carried out to attempt to explain the measured conduction behaviour of such composites, as summarised in [3]. A number of the models developed attempt to determine the probability of conducting particles forming chains across the composite and to relate these probabilities to the volume fraction of conducting particles. This generally leads to the concept of a "percolation threshold', this being the minimum volume fraction required for there to be one or more current paths through the mixture. Above the percolation concentration, the resistivity depends on the number of paths through the material and the resistivity of the conductive filler. A gradually decreasing resistance is predicted for increased filler concentration. For the screen printed ink samples described above the volume fraction is of the order of $13 \%$ while for a similar system using lithographically printed ink the volume fraction is only $3 \%$ for a $200 \mu \mathrm{m}$ thick substrate. It is assumed that following curing the ink is almost entirely made up of conducting particles, thus the volume fraction is just the ink volume as a proportion of the total volume, the ink carrier volume being assumed insignificant. At the low volume fraction available for lithographically printed substrates most of the models predict unacceptably high resistances although, for very thin substrates, acceptable resistance are predicted. Generally these models assume spherical conducting particles while the ink, in its printed state is made up of plates or flakes of silver. The model of Scarisbick [4] shows that plate shaped conducting particles give considerable reductions in resistivity. However care must be taken to ensure that the laser heating is not sufficient to melt the filler particles as this would allow them to become spherical, the worst shape possible for effective conduction through a mixture.

In reality, both of the mechanisms described above have some effect and indeed the reduction in substrate volume resulting from the vaporisation of volatile components will have the effect of increasing the volume fraction of conductive elements in the resulting via.

\section{Via Production Rates}

The production rates which can be achieved using the two proposed methods depend, to some extent, on the lasers available. The drilling of via holes in the materials considered requires of the order of $5 \mathrm{CO}_{2}$ laser pulses at a fluence of $6 \mathrm{Jcm}^{-2}$. Given that standard laser technology offers a repetition rate of around $50 \mathrm{~Hz}$, this implies a drilling time of $100 \mathrm{~ms}$ per via. Allowing for reasonable times for movement of the substrate between vias, this implies a production rate of around 10000 vias per hour. These are similar to the rates claimed for commercial drilling machines for conventional substrates. The production rates achievable using the melt and flow technique will depend heavily on the substrate thickness. In thin materials the greater absorption depth of the $\mathrm{CO}_{2}$ laser means that a large proportion of the depth is directly heated while for thicker material heating is more dependant on the thermal diffusivity. By a similar argument, thinner materials will allow smaller diameter vias to be produced.

\section{Conclusions}

Some of the difficulties invovled in forming vias in lithogrpahically printed circuit substrates have been discussed and two new methods proposed to overcome these problems. The success of these methods has been shown to be dependent on an understanding of a number of material characteristics and the critical parameters have been presented. In particular, 
the laser requirements for the formation of straight and tapered via holes suitable for lithogrpahic ink filling have been presented for Teslin and Glossart substrates. Although excimer lasers will produce more precise and smaller. via holes, the $\mathrm{CO}_{2}$ laser will allow higher production rates. It has been shown that although some filling does occur in straight holes successful via formation will require further development including the use of tapered holes.

It has also been shown that a $\mathrm{CW} \mathrm{CO}$ laser is likely to be the most appropriate for the formation of melt and flow vias due to its greater absorption depth and higher ablation threshold.

\section{Acknowledgments}

The Authors wish to thank Mr M. Grandis of Polyflex for the provision of material samples, Prof. P.E. Dyer for allowing use of facilities and for insight into results, Mr G. Robinson for help in the preparation of SEM images and Mr C. Bartles for work on thermal diffusivity measurements. This work is supported by EPSRC under grant GR/L98664.

\section{References}

1 Ramsey, BJ, Evans, PSA, \& Harrison, D, 'A Novel Circuit Fabrication Technique Using Offset Lithography' Journal of Electronics Manufacture, 1997, Vol. 7, No. 1, pp. 63-67.

2 Shepherd, PR, Evans, PSA, Ramsey, BJ; \& Harrison, D, 'Lithographic Technology for Microwave Integrated Circuits' Electronics Letters, Vol. 33, No. 6, pp. 483-484.

3 Lux, F, "Models Proposed to Explain the Electrical Conductivity of Mixtures Made of Conductive and Insulating Materials', Journal of Material Science, Vol. 28, pp. 285-301, 1993.

4 Scarisbrick, RM, 'Electrically Conducting Mixtures', Journal of Physics D: Applied Physics, Vol. 6, pp. 20982110, 1973.

5 Parker, WJ, Jenkins, RJ, Butler, CP and Abbott; GL, 'Flash Method of Determining Thermal Diffusivity, Heat Capacity and Thermal Conductivity', Journal of Applied Physics, Vol. 32, No. 9, pp. 1679-1684, 1961. 